\title{
Optimization of Directional Sensor Orientation with Application to Sun Sensing
}

\author{
John C. Springmann* and James W. Cutler t $^{\ddagger}$ \\ University of Michigan, Ann Arbor, Michigan 48109
}

\begin{abstract}
DOI: $10.2514 / 1.61468$
A method is presented to optimize the orientation of directional sensors and instruments in a vehicle body-fixed frame. Directional dependence is included by creating a uniformly distributed set of directions in the body-fixed frame and formulating the objective as a function of these directions. The method is demonstrated by application to photodiodes for sun sensing, for which the covariance of the sun vector estimate is derived as a function of the photodiode configuration. The measured sun vector angular accuracy is then minimized as a function of the configuration, which enables the most accurate sun sensing with the given hardware. This technique maximizes subsystem performance and provides a design method to replace traditional, iterative design approaches to sensor placement.
\end{abstract}

\section{Introduction}

A METHOD is presented to optimize the orientation of directional sensors in a vehicle body-fixed coordinate system. This optimization technique can be used in spacecraft design to maximize the performance of directional sensors and instruments. The method is demonstrated by application to photodiodes used for sun sensing in spacecraft attitude determination, where the accuracy of the sun vector estimate is dependent on the orientation of each individual photodiode.

Photodiodes, also referred to as cosine detectors [1], are a common method of sun sensing on small spacecraft because of their simplicity and low cost (for example, [2-6]). Multiple photodiodes can be combined to estimate the line-of-sight vector to the sun, which is subsequently used for attitude determination or instrument pointing. The uncertainty of the estimated sun vector depends on both the performance of the individual photodiodes as well as the orientation of each sensor. In this paper, the covariance of the sun vector estimate is derived as a function of the photodiode configuration, and the orientation of the sensors is optimized to minimize the angular uncertainty of the resulting sun vector estimate.

The traditional spacecraft design process relies on iterating to satisfy design requirements and constraints [7]. In the design process, sensor placement is typically performed manually based on prior experience and similarity to previous designs [8]. Although optimization is becoming more prevalent in spacecraft design $[\underline{9}, 10]$, there are very few methods for the optimization of directional sensors in the literature. In the only paper found on optimal sun sensor configurations, Jackson and Carpenter optimize the orientation of sun sensors by parameterizing the orientation as placement of the sensors on a spherical spacecraft and using genetic algorithms and simulated annealing to maximize coverage of the sensors over the spacecraft body frame [8]. In this work, the angular uncertainty of the sun vector estimates are minimized subject to the orientation of the sun sensors, rather than maximizing the sun sensor coverage, which results in the best attitude determination accuracy with the given hardware. The formulation is general and can be used for the

Presented as Paper 2013-0479 at the 23rd AAS/AIAA Space Flight Mechanics Meeting, Kauai, HA, 10-14 February 2013; received 15 December 2012; revision received 8 July 2013; accepted for publication 1 September 2013; published online 11 March 2014. Copyright $\odot 2013$ by John C. Springmann and James W. Cutler. Published by the American Institute of Aeronautics and Astronautics, Inc., with permission. Copies of this paper may be made for personal or internal use, on condition that the copier pay the $\$ 10.00$ per-copy fee to the Copyright Clearance Center, Inc., 222 Rosewood Drive, Danvers, MA 01923; include the code 1533-3884/14 and $\$ 10.00$ in correspondence with the CCC

*Ph.D. Candidate, Department of Aerospace Engineering; jspringm@ umich.edu.

${ }^{\dagger}$ Assistant Professor, Department of Aerospace Engineering; jwcutler@ umich.edu. optimization of various directional sensors or instruments in the body-fixed frame.

The orientation optimization problem is formulated using a unit sphere. With the spacecraft located at the center, the surface of the sphere represents all directions in the body-fixed coordinate system. This sphere is commonly referred to as the attitude sphere or spacecraftcentered celestial sphere. Directions in the body-fixed frame are created by discretizing the attitude sphere, and these directions are used in the objective function. Directions on the attitude sphere are weighted to account for vehicle- and mission-specific parameters such as orbital inclination and attitude. The nature of the objective function is problem specific and can be minimized using a suitable algorithm. The unique contributions of this paper are the formulation for optimization that uses the attitude sphere as well as application to photodiodes to minimize the uncertainty of the measured sun vector. This new formulation enables the application of existing minimization techniques to a broad range of directional-dependent problems.

The remainder of this paper is organized as follows. In Sec. II, the attitude sphere is discretized to create the directions used to formulate the objective function. The objective function for the sun sensor optimization problem is derived in Sec. III and applied to example design problems in Sec. IV. The method is summarized for general application in Sec. $\underline{V}$ and conclusions are given in Sec. VI.

\section{Use of the Attitude Sphere}

A unit sphere can be used to define all possible directions from the spacecraft to an object of interest in the body-fixed frame. This is referred to as the attitude sphere. This sphere is also commonly referred to as the spacecraft-centered celestial sphere [11]. The attitude sphere is discretized, or pixelized, to create directions over the entire body frame for use in formulating the objective function. Points on the sphere represent the end of unit vectors originating at the center of the sphere.

A pixelization with constant angular resolution over the entire attitude sphere should be used so that portions of the sphere are not artificially weighted due to a higher density of points in the region. Because of this, the typical method of varying the azimuth and elevation evenly to create points on a sphere is not used; it results in a distribution with higher density near the poles, as seen in Fig. 1a. Uniform pixelization of the surface of a sphere is not a new problem. It has been used for mapping the celestial sphere for astronomical applications [12] as well as mapping Earth for remote sensing applications [1] . Attitude sphere pixelization has also been used to formulate path planning for spacecraft attitude control [14].

To pixelate the attitude sphere, the icosahedron-based approach of Tegmark [12] is used because it produces a near-uniform distribution

FFORTRAN code for implementation is available from the author at http:// space.mit.edu/home/tegmark/icosahedron.html [retrieved 12 September 2012]. 


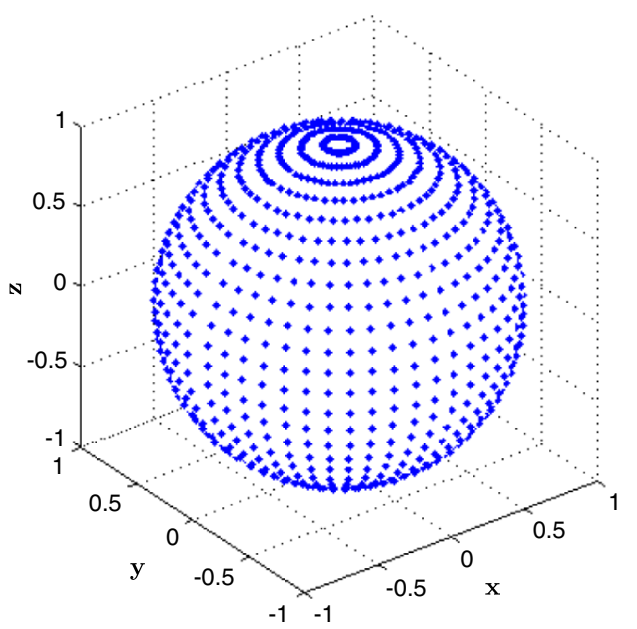

a) Unit sphere with points generated using constant azimuth and elevation increments

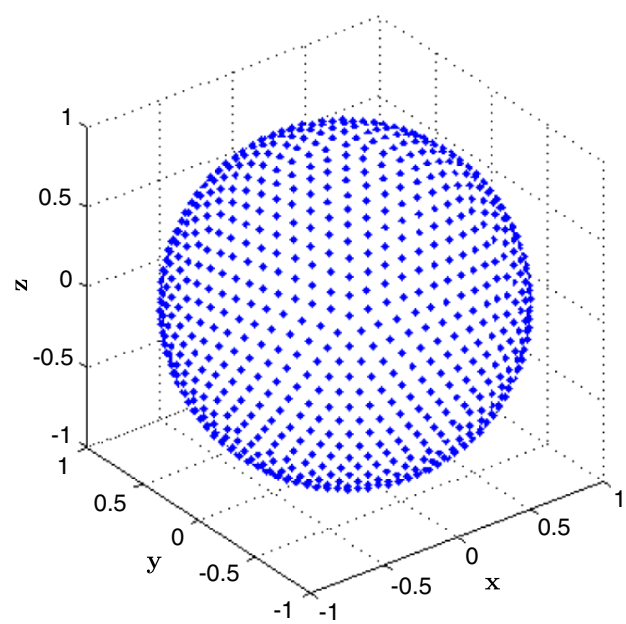

c) Unit sphere with points evenly distributed over the surface using the icosahedron approach

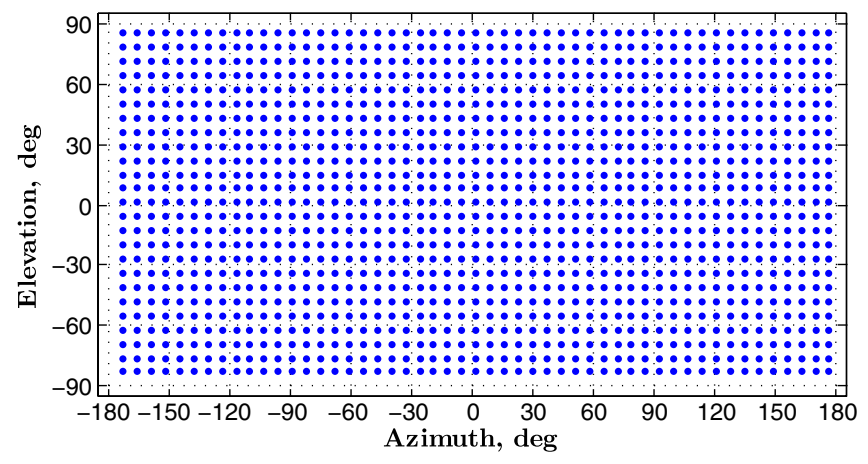

b) 2-D projection of fig $1 \mathrm{a}$

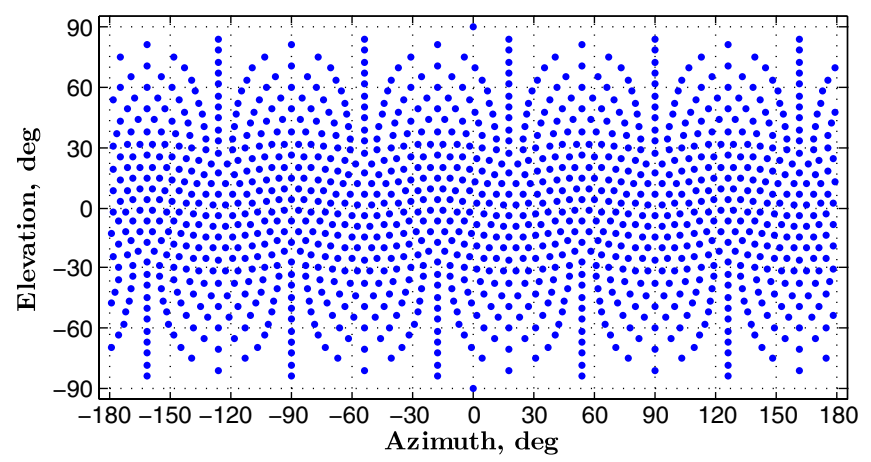

d) 2-D projection of fig $1 \mathrm{c}$

Fig. 1 Unit spheres with two different methods of distributing points shown with their two-dimensional (2-D) projections.

of points on the sphere, as seen in Fig. 1c. The pixelization method begins by inscribing an icosahedron inside a unit sphere. Points are then distributed evenly on each triangular face of the icosahedron. This is shown in Fig. 2. Each point in Fig. $2 b$ is the center of the hexagonal pixel. The pixel centers are then projected from the faces of the icosahedron onto the sphere and shifted slightly to give all pixels approximately equal area.

It is not possible to place an arbitrary number of pixels on the sphere while maintaining the near-uniform distribution. The number of pixels on the sphere is given by

$$
N=40 r(r-1)+12
$$

where $r=1,2,3, \ldots$ is the resolution. A plot of the number of pixels $N$ as well as the average angular distance between pixel centers is shown in Fig. $\underline{3}$.

\section{Formulating the Objective Function for Sun Sensor Optimization}

An ideal photodiode produces current $I$ as a function of incoming light according to the model [15]

$$
I=I_{0} \cos (\theta)
$$

where $\theta$ is the angle between the direction normal to the photosensitive plane and the line-of-sight vector to the sun (herein referred to as the sun vector), and $I_{0}$ is the maximum current output of the sensor, corresponding to $\theta=0$.

Multiple photodiodes can be combined to provide an estimate of the sun vector. The uncertainty of the sun vector estimate is a function of the performance of the individual photodiodes as well as the photodiode configuration. In this section, the objective function to minimize the angular uncertainty of the sun vector estimate is formulated as a function of the photodiode orientation. In Sec. III.A, the covariance of the sun vector estimate is derived, and this is used with the directions on the attitude sphere to formulate the objective function in Sec. III.B.

\section{A. Sun Vector Estimation from Multiple Photodiodes}

A single photodiode provides one dimension of sun vector information; multiple photodiodes can be combined to provide a full sun vector. Two common photodiode configurations are to use six photodiodes that are orthogonal [2,3] or to place pairs of photodiodes angled in a single plane [1]. The former method does not provide photodiode coverage sufficient for sun vector estimation over the entire attitude sphere because the field of view of individual photodiodes is generally less than $180 \mathrm{deg}$. In the latter configuration, the two photodiode readings can be used to estimate the sun vector component in the common plane of the photodiodes, thus achieving a one-axis sun measurement [1]. For unconstrained optimization, a method to estimate the sun vector from an arbitrary photodiode configuration is required, which is derived in this section. 


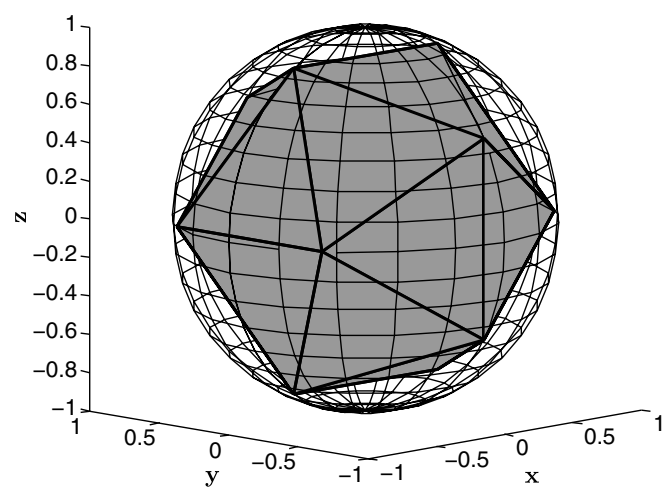

a) An icosahedron with a unit sphere overlaid

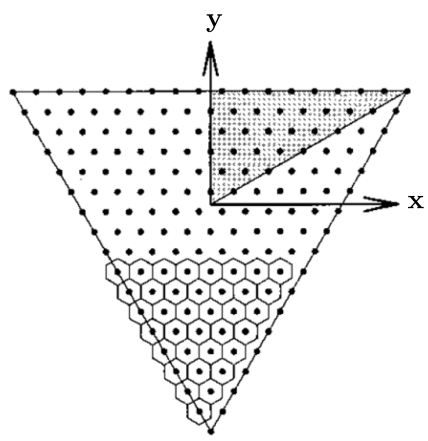

b) The triangular icosahedron faces are pixelated with a regular triangular grid. The points shown are the centers of the hexagonal pixels

Fig. 2 First two steps of the icosahedron-based approach to sphere pixelization are shown. Figures adapted from [12]

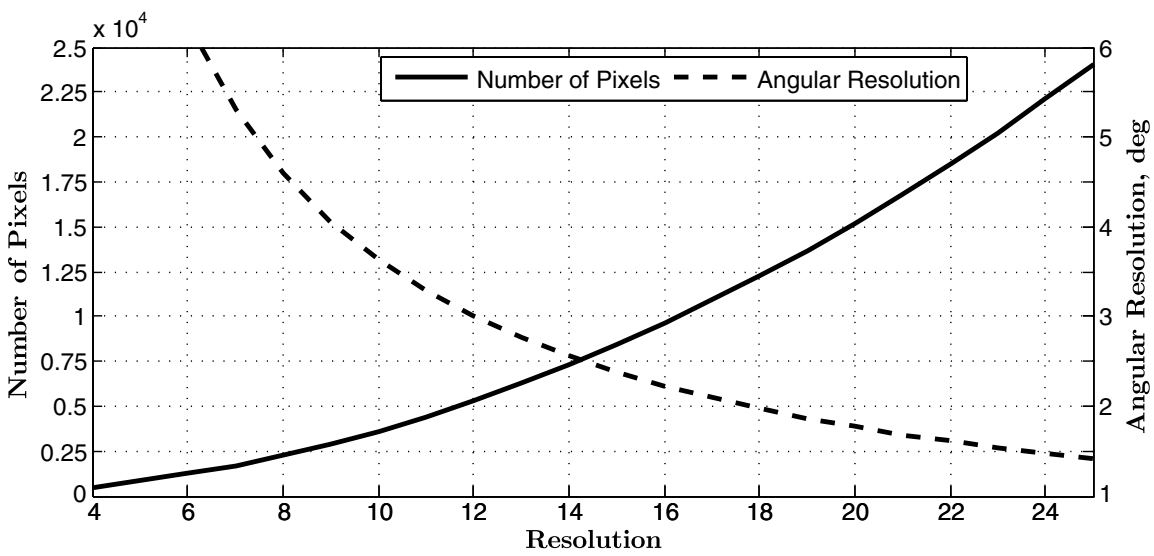

Fig. 3 Number of pixels $N$ and corresponding angular resolution versus the resolution $r$.

The sun vector estimation is formulated as finding the intersection of multiple planes, where the planes are defined by the photodiode normal directions and the measured currents. Intersection of planes is used rather than intersection of cones to estimate the sun vector from the photodiode measurements because the former is a linear function of the photodiode measurements. The formulation is illustrated for a two-dimensional case in Fig. 4. In the figure, the rectangles represent two photodiodes, the dashed arrows show the directions normal to the photosensitive plane $\hat{\boldsymbol{n}}_{i}$ (where $i \in\{1,2\}$ is the photodiode index), the solid arrow shows the sun vector $\hat{\boldsymbol{s}}$, and $\theta_{i}$ is the angle between the photodiode normal directions and the sun vector, as in Eq. (2). The sun vector $\hat{\boldsymbol{s}}$ corresponds to the intersection point of the two planes, shown by the dotted lines, and $\hat{\boldsymbol{s}}$ is estimated by finding the intersection point of the two planes. The planes are perpendicular to the photodiode normal direction, and, referring to Eq. (2), their location along the normal direction $\hat{\boldsymbol{n}}_{i}$ is $I_{i} / I_{0, i}$.

In general, a plane can be defined by a known point on the plane $\boldsymbol{r}_{0}$ and a normal vector to the plane $\boldsymbol{p}$, according to Eq. (3), where $\boldsymbol{r}$ is the location of any point on the plane:

$$
p^{T}\left(r-r_{0}\right)=0
$$

In application to the photodiode configuration of Fig. $\underline{4}$, Eq. (3) becomes

$$
\hat{\boldsymbol{n}}_{i}^{T}\left(\hat{\boldsymbol{s}}-\frac{I_{i}}{I_{0, i}} \hat{\boldsymbol{n}}_{i}\right)=0
$$

With $\hat{\boldsymbol{n}}_{i}=\left[\begin{array}{lll}n_{i, 1} & n_{i, 2} & n_{i, 3}\end{array}\right]^{T}$ and $\hat{\boldsymbol{s}}=\left[\begin{array}{lll}s_{1} & s_{2} & s_{3}\end{array}\right]$, and using the fact that $\|\hat{\boldsymbol{n}}\|=1$, Eq. (4) can be rewritten as

$$
\frac{I_{i}}{I_{0, i}}=\left[\begin{array}{lll}
n_{i, 1} & n_{i, 2} & n_{i, 3}
\end{array}\right]\left[\begin{array}{c}
s_{1} \\
s_{2} \\
s_{3}
\end{array}\right]
$$

Equation (5) can also be obtained directly by rewriting Eq. (2) as $I=I_{0} \cos (\theta)=I_{0} \hat{\boldsymbol{n}}^{T} \hat{\boldsymbol{s}}$, but derivation with Fig. 4 is included to provide further insight into the vector measurement formulation. For sun sensing in three dimensions, at least three nonparallel and noncoplanar photodiodes are required. With photodiodes satisfying these conditions, the sun vector corresponds to the intersection point of the planes from each photodiode.

In practice, a single intersection point generally does not exist due to measurement error. The ideal model of Eq. (2) is corrupted by

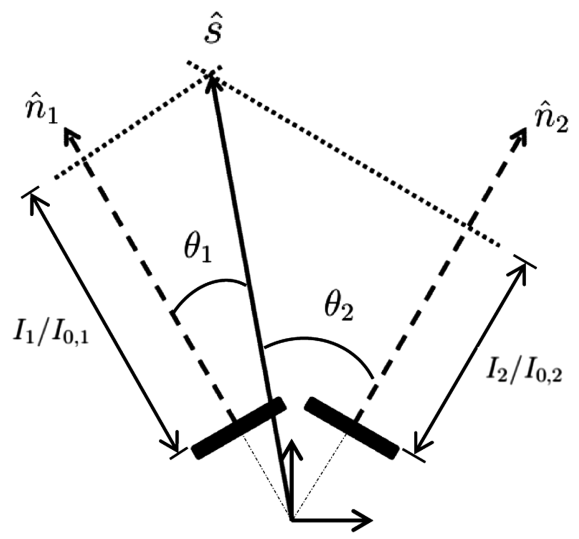

Fig. 4 Illustration of two photodiodes in a single plane. 
sensor noise, and the measurement model is given by Eq. (6), where $\tilde{I}$ is the measured current, and $\eta$ is zero-mean measurement noise:

$$
\tilde{I}=I_{0} \cos (\theta)+\eta
$$

Given at least three nonparallel and noncoplanar photodiodes, the sun vector estimate is the solution to

$$
y=H s-\eta
$$

where, from Eq. (),

$$
\boldsymbol{y}=\left[\begin{array}{c}
\tilde{I}_{1} \\
I_{0,1} \\
\tilde{I}_{2} \\
I_{0,2} \\
\vdots \\
\tilde{\tilde{I}}_{k} \\
\hline I_{0, k}
\end{array}\right], \quad H=\left[\begin{array}{ccc}
n_{1,1} & n_{1,2} & n_{1,3} \\
n_{2,1} & n_{2,2} & n_{2,3} \\
\vdots & \vdots & \vdots \\
n_{k, 1} & n_{k, 2} & n_{k, 3}
\end{array}\right], \quad \eta=\left[\begin{array}{c}
\frac{\eta_{1}}{I_{0,1}} \\
\frac{\eta_{2}}{I_{0,2}} \\
\vdots \\
\frac{\eta_{k}}{I_{0, k}}
\end{array}\right]
$$

and $k$ is the number of photodiodes illuminated. This is the classic linear least-squares problem. Using maximum likelihood estimation, the best estimate of $s$ is found by minimizing the objective function given by Eq. (9) with measurement covariance $R=\mathbb{E}\left(\eta \eta^{T}\right)$. . From the Gauss-Markov theorem, the unconstrained optimal estimate is given by Eq. (10), and the covariance of the estimate is given by Eq. (11) [17]:

$$
\begin{gathered}
J\left(\boldsymbol{s}_{\mathrm{est}}\right)=\frac{1}{2}\left[\boldsymbol{y}-H \boldsymbol{s}_{\mathrm{est}}\right]^{T} R^{-1}\left[\boldsymbol{y}-H \boldsymbol{s}_{\mathrm{est}}\right] \\
\boldsymbol{s}_{\mathrm{est}}=\left(H^{T} R^{-1} H\right)^{-1} H^{T} R^{-1} \boldsymbol{y} \\
P=\left(H^{T} R^{-1} H\right)^{-1}
\end{gathered}
$$

The sun vector estimate resulting from the linear least-squares solution of Eq. (10) is not constrained to be a unit vector, and the covariance $P$ is full rank. For an optimal unit vector estimate, the objective function $J\left(s_{\text {est }}\right)$ should be minimized with the nonlinear constraint $\boldsymbol{s}_{\mathrm{est}}^{T} \boldsymbol{s}_{\mathrm{est}}=1$. The constrained minimization can be carried out with various numerical techniques. Alternatively, a unit vector estimate can be obtained through brute-force normalization of Eq. (10), but while the angular difference between this and the optimal unit vector estimate may be negligible for a given photodiode configuration, the difference can be on the order of tenths of degrees depending on the photodiode configuration. Therefore, constrained minimization of the objective function should be used.1

The covariance $P$ is that of the unconstrained solution. The covariance of a unit vector is necessarily rank deficient and can be difficult to calculate. A covariance approximation commonly used for attitude determination sensors is the QUEST measurement model, for which the covariance of a measured unit vector $\hat{\boldsymbol{u}}$ is given by

$$
R_{\mathrm{QMM}}=\sigma^{2}\left(I_{3 \times 3}-\hat{\boldsymbol{u}} \hat{\boldsymbol{u}}^{T}\right)
$$

where $\sigma^{2}$ is the variance of the components normal to the estimated direction [19]. For triaxial sensors, an assumption of uniform

\footnotetext{
${ }^{\S}$ It is common to assume the uncertainty in each photodiode has a Gaussian distribution, and this assumption is used in the simulations presented in this paper. In practice, characteristics of the sensor should be measured by calibration.

'One drawback of the constrained minimization is that any errors in the photodiode parameters (their orientation and maximum current output) will not be evident. When working with flight data, the magnitude of the unconstrained sun vector estimate is a useful verification of the calibration parameters. Even the unconstrained estimate should yield a vector with unit magnitude on average, and a consistently biased vector magnitude indicates that sensor calibration is required [18].
}

uncertainty in all directions is typically valid, and so $\sigma^{2}$ can be taken to be the variance in one of the sensor axes. This approximation is not applicable to photodiodes in arbitrary configurations because the uncertainty in each direction is not equal and depends on the photodiode configuration. This is quantified by the vector covariance $P$ of Eq. (11).

The covariance of the optimal unit vector estimate can be approximated by the linearized transformation of the unconstrained covariance that is equivalent to brute-force normalization of the unconstrained sun vector estimate. That is, the covariance of the unit vector estimate $P^{\prime}$ is given by Eq. (13), where $f(\boldsymbol{x})$ is given by Eq. (14) and the subscript $\boldsymbol{x}=\hat{\boldsymbol{s}}_{\mathrm{est}}$ indicates that the Jacobian is evaluated at the optimal unit vector estimate:

$$
\begin{gathered}
P^{\prime}=\left.\left.\frac{\partial f}{\partial \boldsymbol{x}}\right|_{x=\hat{s}_{\mathrm{est}}} ^{T} P \frac{\partial f}{\partial \boldsymbol{x}}\right|_{\boldsymbol{x}=\hat{s}_{\mathrm{est}}} \\
\hat{\boldsymbol{x}}=f(\boldsymbol{x}) \equiv \frac{\boldsymbol{x}}{\sqrt{\boldsymbol{x}^{T} \boldsymbol{x}}}
\end{gathered}
$$

\section{B. Formulation of the Objective Function to Minimize Angular Uncertainty}

From Eqs. (11) and (13), the sun vector uncertainty is a function of the sensor noise, manifested in $R$, and the orientation of each illuminated photodiode, manifested in $H$. The goal of the optimization is to determine the photodiode normal directions that minimize the unit sun vector covariance $P^{\prime}$. The photodiode normal directions (originating at the center of the attitude sphere, which coincides with the center of the body-fixed frame) are parameterized by their azimuth $\alpha$ and elevation $\beta$ in the spacecraft body-fixed frame, and it is assumed the measurement covariance is known. To optimize the configuration over the entire attitude sphere, the sum of the weighted covariances of each direction on the attitude sphere is minimized. The objective function is

$$
J=\sum_{j=1}^{N} W_{j} \operatorname{tr}\left(P_{j}^{\prime}(\boldsymbol{\alpha}, \boldsymbol{\beta})\right)
$$

where $j$ is the index of the direction on the attitude sphere, $N$ is the total number of directions on the sphere [Eq. (1)], $W_{j}$ is the weight of the $j$ th direction, $P_{j}^{\prime}$ is the unit sun vector covariance matrix given by Eq. (13) when the sun is in the $j$ th direction, $\alpha$ and $\boldsymbol{\beta}$ are column vectors of the azimuths and elevations of the photodiode normal directions, and $\operatorname{tr}(\cdot)$ denotes the trace of a matrix. $W_{j}$ is discussed in the examples of Sec. IV. $P_{j}$ depends on which photodiodes are illuminated for the $j$ th sun vector direction; only the rows of $H$ and $R$ that correspond to illuminated photodiodes are used in the calculations.

\section{Application}

The formulation of Sec. III is applied to optimize the photodiode configuration in design examples. This demonstrates the optimization method and shows its utility in providing a design technique to maximize performance of sensors. In Sec. IV.A, the utility of weighting in the objective function is discussed, and the optimization technique is applied to two design examples in Sec. IV.B.

\section{A. Weighting the Attitude Sphere}

The directions on the attitude sphere have been used to formulate the objective function. In some applications, certain directions are more important than others. For example, Earth-facing regions of the attitude sphere for an Earth-pointing spacecraft will never contain a sun vector direction during nominal operations, thus those directions would carry less weight in the objective function. To account for this in the optimization, unique weights can be assigned to each direction. These weights are given by $W_{j}$ in Eq. $(\underline{15})$. For spacecraft, the 
weighting is a function of the expected orbital parameters and spacecraft attitude.

As an example application, consider a nadir-pointing spacecraft in a $400 \mathrm{~km}, 90 \mathrm{deg}$ inclination circular orbit. The spacecraft attitude is such that the body $z$ axis is aligned with the nadir direction and the rotation rate is $1 \mathrm{deg} / \mathrm{s}$ in the $z$ axis. The relative importance of the $j$ th direction on the attitude sphere for sun sensor optimization can be quantified by the amount of time that the sun vector is expected to be in the $j$ th direction. To determine these times, the spacecraft was simulated for a one year mission using Analytical Graphic, Inc.'s Systems Tool Kit.- In the simulation, the sun vector resolved in the spacecraft body frame was polled once every $4 \mathrm{~s}$, and after eliminating data points in eclipse, this results in 5,343,459 sun vectors, which are subsequently binned to the nearest direction on the attitude sphere.

Figure 5 shows the number of occurrences of the sun vector at every direction on the attitude sphere (directions with zero occurrences are not shown). In this example, 2892 directions on the attitude sphere are used for an average angular resolution of $4 \mathrm{deg}$. The simulated spacecraft spin rate and sun vector sampling frequency results in the sun vector being polled after approximately 4 deg of spacecraft rotation, which is sufficient for the angular resolution of the attitude sphere. In photodiode orientation optimization for a mission with this orbit and attitude, the relative weighting shown in Fig. 5 can be used to improve the accuracy of the sun vector estimates for the most common sun vector directions expected during the mission. The most common sun vector direction for this scheme is at an elevation of $-25 \mathrm{deg}$ in the body-fixed frame, and regions above an elevation of $30 \mathrm{deg}$ have zero sun vector occurrences.

\section{B. Design Examples}

With identically weighted directions on the attitude sphere, there are an infinite number of optimal configurations for a given number of photodiodes. In practice, the configuration will be constrained by factors such as a lack of available surface area on the spacecraft to place sensors (due to solar cells, instruments, etc.) or orientations that are not useful due to shadowing by other spacecraft components. These factors should be used to provide an initial feasible configuration to be optimized. In this section, two examples demonstrating the procedures and potential utility of the optimization method are provided, and remarks on global versus local solutions are given at the end of the section. In both examples, the photodiodes have a conical field of view of $140 \mathrm{deg}$, a maximum current output of one, and a measurement uncertainty with a standard deviation of 0.02 . These parameters are representative of sensors used by the authors on past missions.

\section{Example 1}

Consider a cubical spacecraft on which, given the constraints of the telemetry system, it is possible to place two photodiodes on each face of the cube. This results in 24 design variables (azimuth and elevation for each sensor). As a first step in the optimization process, the number of design variables is first reduced to explore the solution space and determine an initial condition for optimization that meets design constraints. The problem is reduced to a single design variable by using the geometry of Fig. $\underline{6}$, where the elevation of each sensor relative to the surface to which it is mounted $\gamma$ is the single design variable. The objective value as a function of the elevation angle is shown in Fig. 7a. The elevation angle has been varied over the entire range, which maintains photodiode coverage sufficient for sun vector estimation over the entire attitude sphere ( $4 \pi$ steradian coverage). Two weighting schemes are used and shown in Fig. 7: identical weighting, where each direction on the attitude sphere is weighted equally, and nadir weighting, which is the weighting example given in Sec. IV.A. The former weighting would be used if the attitude control scheme is not finalized at this time in the design scheme or so that the design is robust to any attitude control scheme, and

**Data available online at http://www.agi.com/products/ [retrieved January 2014].

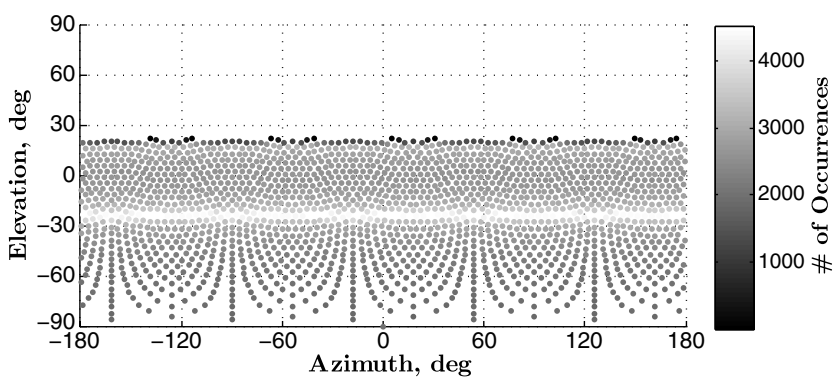

Fig. 5 The directions on the attitude sphere color-coded by the number of occurrences of the sun vector for the nadir-pointing spacecraft example of Sec. IV.A. Directions with zero occurrences are not shown.

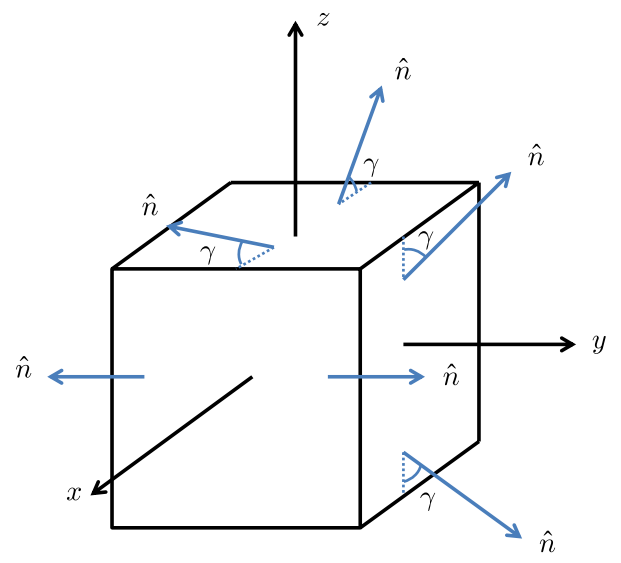

Fig. 6 Photodiode configuration for example 1 reduced to a single design variable. There are two photodiodes per face of the cube and the directions normal to the photosensitive planes are labeled with $\hat{n}$. The photodiodes on the $+x,+y$, and $+z$ surfaces are in the $x-y, y-z$, and $x-z$ planes, respectively, and each photodiode is tilted by angle $\gamma$ from the surface, where $\gamma$ is the single design variable. Photodiodes on the $-x$, $-y$, and $-z$ faces are not shown but have an identical configuration as the opposite faces of the cube.

the nadir weighting would be used if the design was for an Earthpointing spacecraft. The objective values have been normalized by their maximum value from each weighting scheme to facilitate comparison on the same plot. The two different weighting schemes result in different optimal photodiode configurations. The optimal mounting angle for the identical weighting is $57 \mathrm{deg}$, and the optimal angle for the nadir weighting is $47 \mathrm{deg}$.

The total angular uncertainty over the attitude sphere is shown as a function of the elevation angle in Fig. 7b. Calculation of this angular uncertainty is discussed in the Appendix. For this example, varying the elevation angle over the range of angles that maintain $4 \pi$ steradian coverage does not result in a significant improvement in angular uncertainty.

The angles that achieve the minimum objective value shown in Fig. $7 \mathrm{a}$ are optimal given the constraints of the geometry shown in Fig. $\overline{6}$. To improve the configuration, these configurations are used as initial conditions to minimize the objective value with the full 24 design variables. MATLAB's optimization toolboxti is used to minimize the objective function subject to the design variables (fmincon, an algorithm to find the minimum of constrained nonlinear multivariable functions, is used to carry out the minimization). The only constraint imposed during the optimization is to maintain sufficient photodiode coverage for sun sensing over $4 \pi$ steradians.

The initial and optimized photodiode normal directions for both weighting schemes are shown in Figs. $\underline{8 \mathrm{a}}$ and $\underline{8 \mathrm{c}}$, and the angular uncertainty of the optimized configurations are shown in Figs. $\underline{8 \mathrm{~b}}$ and $\underline{8 \mathrm{~d}}$. The effect of the weighting is evident in Fig. $\underline{8 \mathrm{~d}}$, where the

${ }^{\dagger}$ Data available online at http://www.mathworks.com/help/optim/ug/ fmincon.html [retrieved November 2012]. 


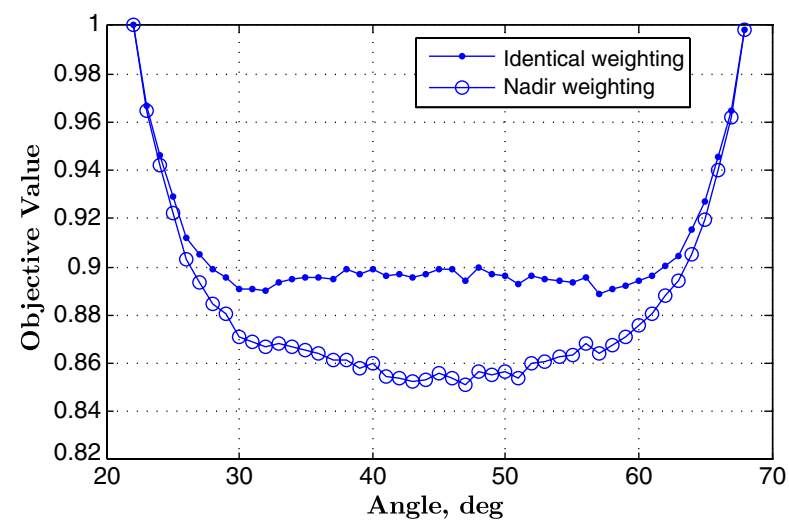

a) Objective value

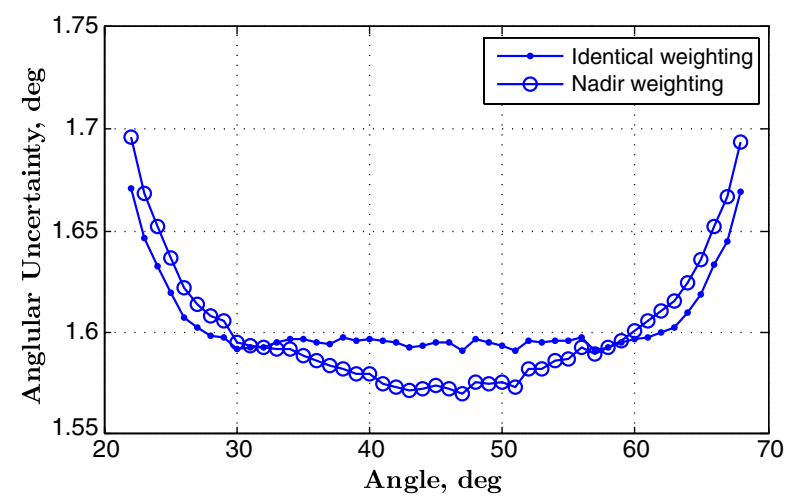

b) Sun vector angular uncertainty

Fig. 7 Objective value and sun vector angular uncertainty as a function of photodiode mounting angle relative to the spacecraft surface in the geometry of Fig. 6. The minimum objective values are achieved with mounting angles of 57 and 47 deg for the identical and nadir-weighting schemes, respectively. Calculation of the sun vector angular uncertainty is discussed in the Appendix.

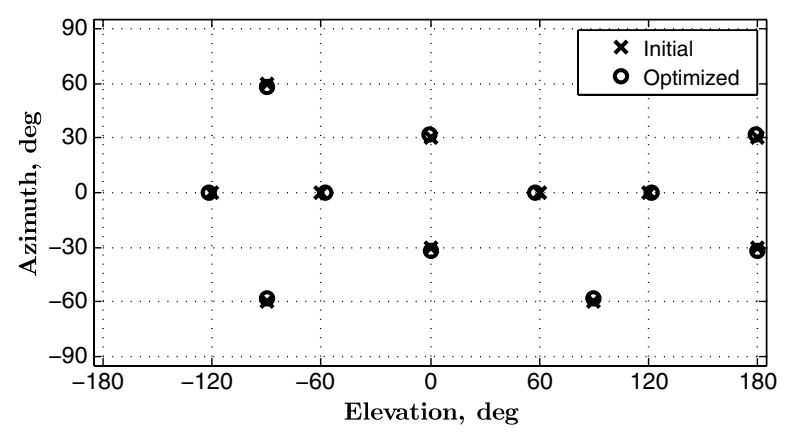

a) Initial and optimized photodiode configurations for the case of identical weighting over the attitude sphere

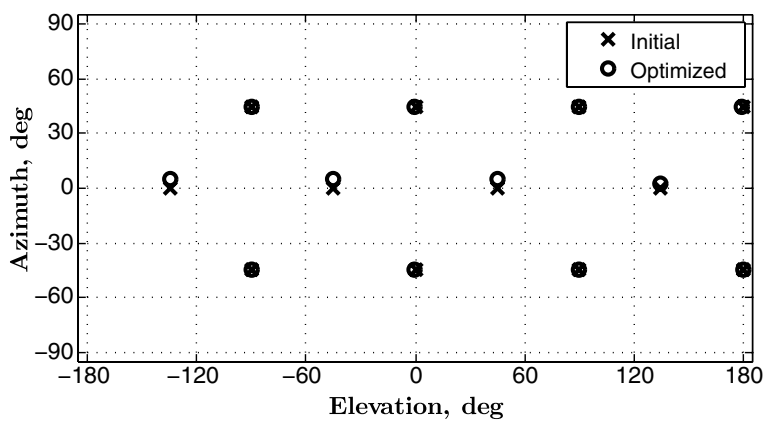

c) Initial and optimized photodiode configurations for the nadir-pointing weighting scheme

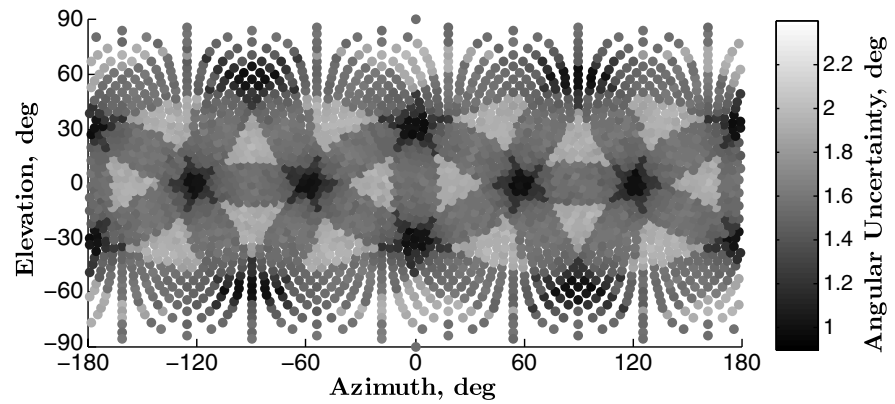

b) Angular uncertainty of the sun vector estimates that result from the optimized configuration for the identical weighting scheme

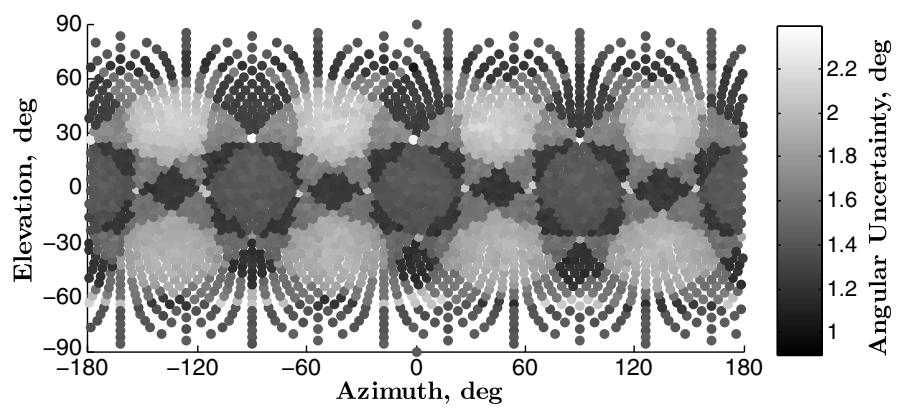

d) Angular uncertainty of the sun vector estimates that result from the optimized configuration for the nadir-pointing weighting scheme

Fig. 8 Photodiode orientations and the resulting sun vector angular uncertainties over the attitude sphere for example 1. In Figs. $\underline{8 a}$ and $\underline{8 c}$, the azimuth and elevation angles are those of the direction normal to the photosensitive plane in the body-fixed frame.

uncertainty in the weighted region (where the elevation is less than $25 \mathrm{deg}$, see Fig. 5) is less than in the region with zero weight. But for the case of identical weighting over the attitude sphere, the uncertainty is mirrored about an elevation of $0 \mathrm{deg}$. For comparison with Fig. $7 \mathrm{~b}$, the total angular uncertainties of the identically and nadir-weighted optimizations are 1.59 and $1.57 \mathrm{deg}$, respectively, which are insignificant improvements over the single variable optimization. The impact of the optimization will vary with the type of problem (number of sensors, design constraints, weighting scheme); example 2 demonstrates the utility of the optimization in achieving significant angular accuracy improvements.

\section{Example 2}

As a second example, consider a cubical spacecraft with the geometry shown in Fig. 9 , which is derived from the actual design constraints of the RAX-2 spacecraft, shown in Fig. 10 [20]. On RAX2 , antennas extend from the $\pm z$ surfaces, potentially shadowing photodiodes mounted to these surfaces. To provide coverage in case of shadows, multiple sensors are placed in different locations with the same orientation. This orientation is normal to the surface; other orientations are not possible due to satellite structural requirements. Three photodiodes per $x / y$ surface is the maximum allowable number of sensors constrained by the number of analog-to-digital converted channels in the telemetry system. These sensors can be tilted relative to the surface to which they are mounted, as in Fig. 10b.

The same procedure as in example 1 is used, where the problem is first reduced to a single design variable to choose feasible initial conditions for the constrained multivariable optimization. The single design variable is the angle of the photodiode normal directions from the surface to which they are mounted, as shown in Fig. 9. The 


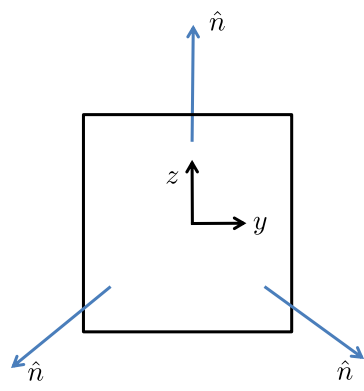

a) $+x$ surface

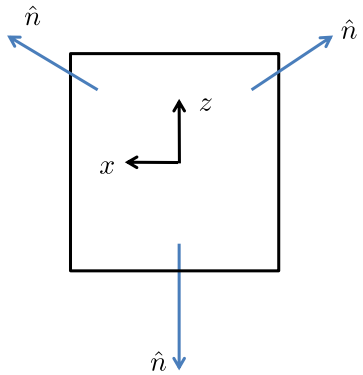

b) $+y$ surface

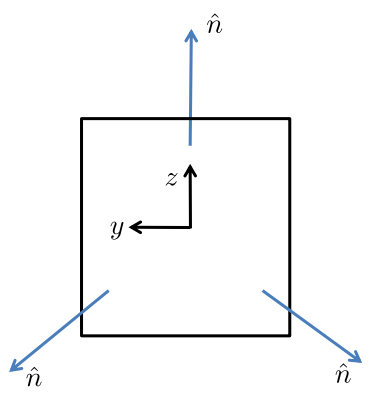

c) $-x$ surface

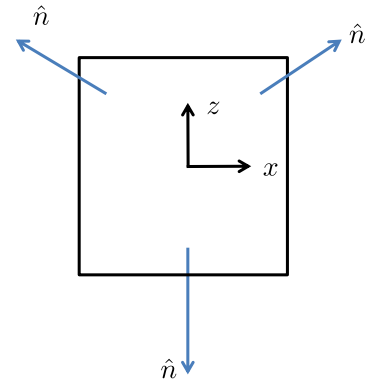

d) $-y$ surface

Fig. 9 Photodiode configuration for example 2 reduced to a single design variable, which is the mounting angle of the photodiodes on the $x / y$ surfaces. Single photodiodes are placed on the $\pm z$ surfaces of a cubical spacecraft and orientated normal to the surface (not shown). The three photodiodes on each of the remaining surfaces are shown with their normal directions denoted by $\hat{n}$. They are oriented in increments of 120 deg in the plane of the surfaces and are tilted from the surfaces (out of the page) by the single design angle.

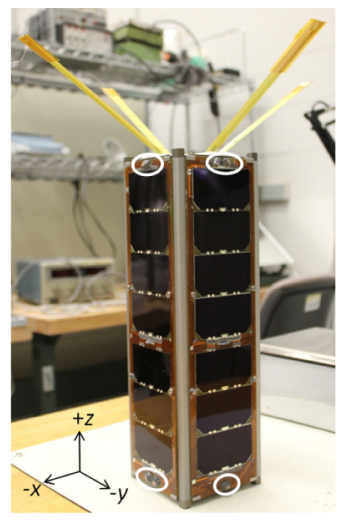

a) RAX-2 flight unit with photodiode locations circled

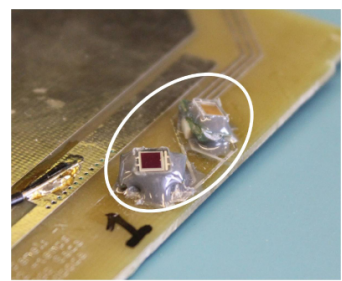

b) Two angled photodiodes located near the $-z$ edge of the $-x$ surface (see coordinate system in fig 10a)

Fig. 10 RAX-2 satellite and photodiodes.

objective values and resulting total sun vector angular uncertainties for both weighting schemes as a function of mounting angle is shown in Fig. 11. The angle was varied over the maximum range that maintained sufficient coverage for sun sensing over $4 \pi$ steradians. In this example, the optimal angle for both weighting schemes is the same. Unlike example 1, the angular accuracy of the estimated sun vector as a function of the photodiode configuration varies significantly. In the nadir-weighting scheme, angular accuracy improvement from 2.4 to $1.7 \mathrm{deg}$ can be achieved by varying the single mounting angle.

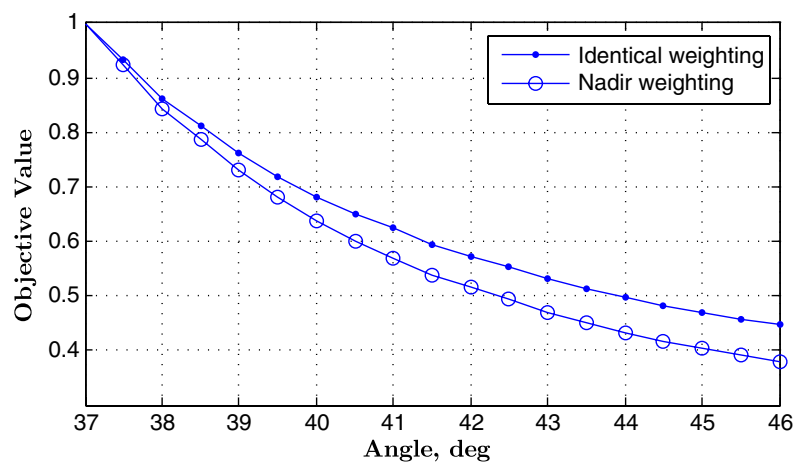

a) Objective value

Fig. 11 Objective value and sun vector angular uncertainty as a function of photodiode mounting angle relative to the spacecraft surface in the geometry of Fig. 9. Minimum objective values are achieved at the same mounting angle for both weighting schemes. Unlike example 1, the angular uncertainty of the estimated sun vector varies significantly over the range of mounting angles that provide $4 \pi$ steradian sun sensing coverage.

The best angle obtained from varying the single design variable is used as the initial condition for the full multivariable optimization problem. MATLAB's fmincon is again used to optimize the configuration subject to 28 design variables (three sensors per $x / y$ surface and one each on the $z$ surfaces). The only constraint imposed during the optimization is that the configuration must provide $4 \pi$ steradian coverage. The resulting optimal orientation of all sensors is shown in Fig. 12a, and the angular uncertainty of the optimal configuration for each weighting scheme is shown in Figs. $12 \mathrm{~b}$ and $12 \mathrm{c}$. The total angular uncertainty of the optimized configurations achieved with both weighting schemes is $1.5 \mathrm{deg}$. Compared with the worst-case accuracy from variation of the single design variable shown in Fig. 11 (2.1 and $2.4 \mathrm{deg}$ ), this is an improvement of 40 and $60 \%$ for the identical and nadir-weighting schemes, respectively. This highlights the utility of optimizing the configuration for maximum angular accuracy rather than simply choosing a configuration with $4 \pi$ steradian coverage.

\section{Global and Local Solutions}

In both examples, the problem was first reduced to single-variable optimization. The single-variable optimal solution was then used as the initial condition for the full multivariable optimization, which was carried out with MATLAB's fmincon, which finds the nearest local minimum and is not a global optimal solution. In the photodiode optimization, there are a large number of solutions with nearly the same objective value (for example, see Fig. 11a), and in practice, there are additional factors that drive the design and provide an initial configuration for optimization. As demonstrated with example 2, finding a local optimum near the initial condition can result in significant accuracy improvements, even if it is not proven to be a globally optimal solution. The approach presented in this paper enables the use of standard minimization techniques for directional

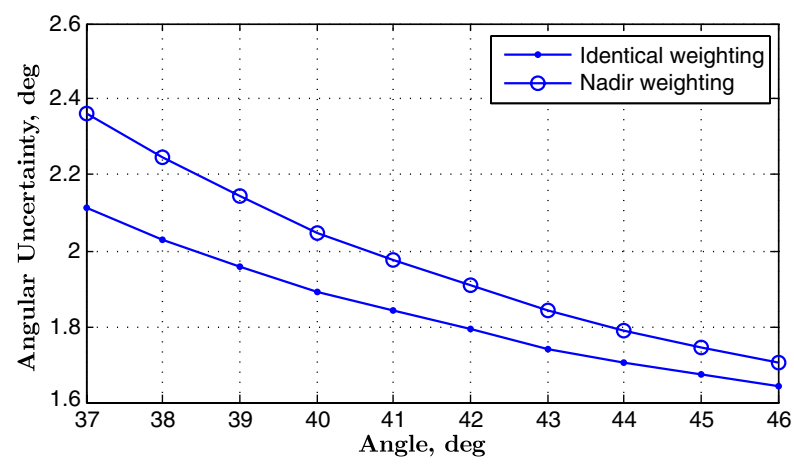

b) Sun vector angular uncertainty 


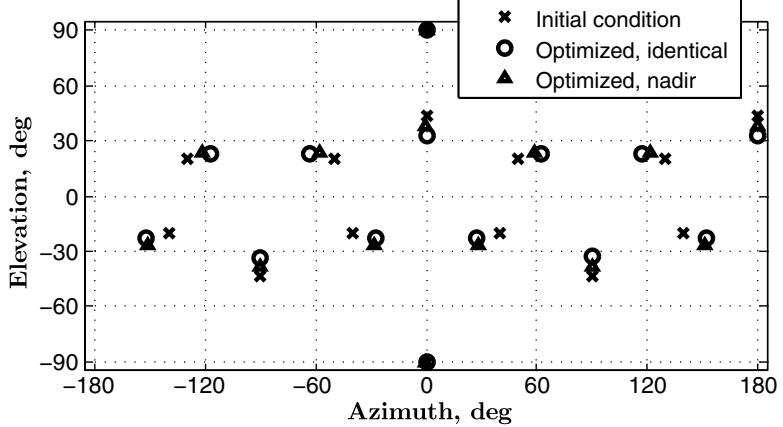

a) Initial and optimized photodiode configurations

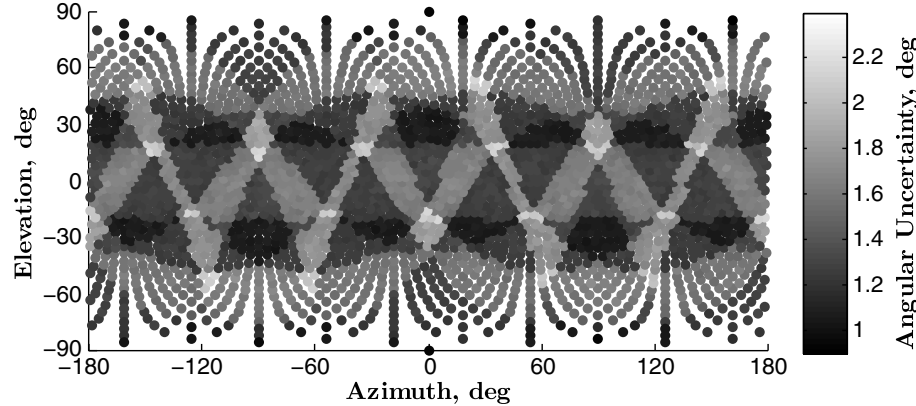

b) Sun vector angular accuracy resulting from the configuration optimized using the identical weighting scheme

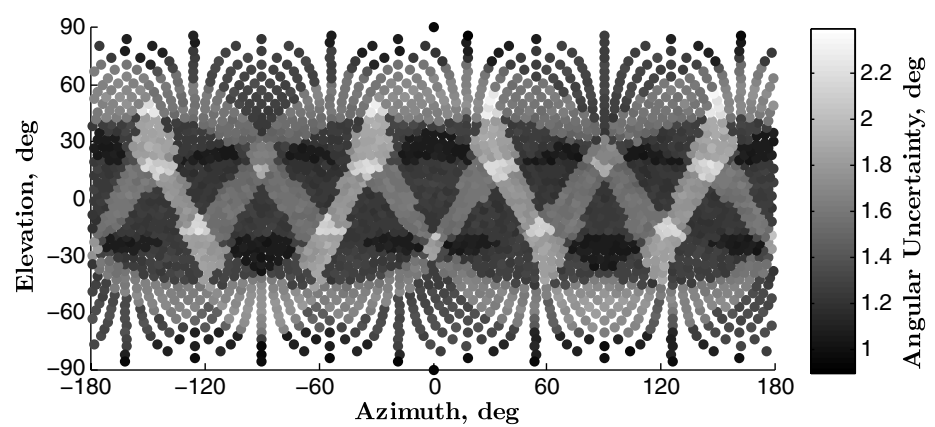

c) Sun vector angular accuracy resulting from the configuration optimized using the nadir-pointing weighting scheme

Fig. 12 Photodiode orientations and the resulting sun vector angular uncertainties over the attitude sphere for example 2. In Fig. 12a, the azimuth and elevation angles are those of the direction normal to the photosensitive plane in the body-fixed frame.

sensor optimization. Techniques such as genetic algorithms or simulated annealing could be used instead of fmincon to find globally optimal solutions, but a detailed discussion on the available optimization techniques and comparison of their results is beyond the intended scope of this paper.

\section{Generalization of the Optimization Method}

The optimization method has been applied to photodiode configurations to minimize the angular uncertainty in sun sensing, but it is generally applicable to optimize the configuration of directional sensors and instruments. Potential additional applications include optimizing the orientations of body-fixed solar panels, antennas, and other types of attitude sensors. Here, the main steps of the optimization method are summarized.

The first step is to discretize the attitude sphere. The attitude sphere represents all directions in the vehicle body-fixed frame, and it is discretized to form a finite set of directions over which to optimize. The discretization is also referred to as pixelization. The icosahedronbased approach to discretization is used because it produces points that have a uniform angular distribution over the sphere [12]. Uniform distribution is important so that regions of the sphere are not artificially weighted by a higher density of points. The user must select the resolution of directions on the sphere based on the application. The relationship between the number of directions on the sphere and angular resolution of the directions is shown in Fig. 3 .

The second step is to formulate the objective function. To do so, the quantity to be optimized must be derived as a function of directions on the attitude sphere and the design variables. In application to photodiodes, the covariance of the sun vector was minimized as a function of photodiode orientation. If the relative importance of the directions on the sphere is not identical, each direction can be given a unique weight in the objective value. This can be used to account for vehicle- or mission-specific parameters, such as orbit and attitude. In the example of Sec. IV.A, dwell time of the sun in each direction of the attitude sphere was used as the weight. Abstracting orbit and attitude as weights is advantageous because, for a given sensor configuration, the component of the objective function in each direction on the attitude sphere needs to be calculated just once and then can be weighted for various potential mission parameters. This facilitates analysis of the robustness of a configuration over different mission parameters.

The final step is to minimize the objective value as a function of the design parameters. The behavior of the objective function will be problem specific. The photodiode optimization example was nonlinear and discontinuous; the problem was first reduced to a single design variable by limiting the configuration. Given that there are an infinite number of optimal photodiode configurations for an identically weighted attitude sphere (free rotation around the sphere is possible), the initial reduction of the problem is useful to provide a feasible configuration that meets design constraints. MATLAB's optimization toolbox was then used to carry out the constrained minimization will the full set of variables to find locally optimal solutions. In general, the user must determine a suitable minimization algorithm based on the specific objective function.

\section{Conclusions}

A method to optimize the orientation of directional sensors in a vehicle body-fixed frame has been presented. The attitude sphere is used to formulate the optimization problem, and vehicle- and mission-specific parameters such as orbit and attitude are accounted for by weighting directions on the sphere. This formulation provides a method generally applicable to directional sensors and instruments.

The utility of the method has been demonstrated by application to photodiodes for sun sensing in attitude determination subsystems, where it was used to find photodiode configurations that minimize uncertainty in the resulting sun vector estimate. This maximizes the performance of the photodiodes used for attitude determination, and an improvement of $60 \%$ in angular accuracy was demonstrated in one of the examples. The method is useful not only because it maximizes the performance of a given set of hardware, but also because it provides a design technique to determine sensor orientation that replaces traditional methods in which placement is manually iterated 


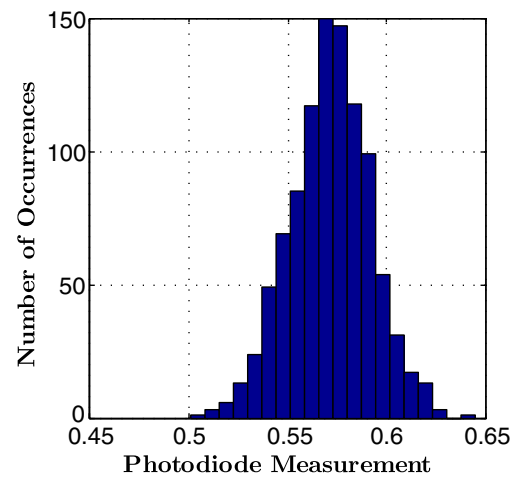

a) Sensor 1

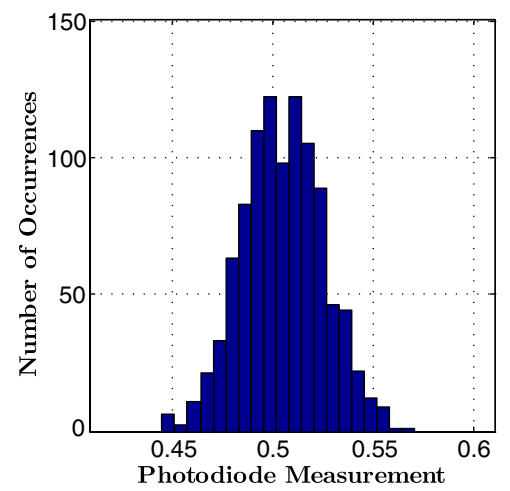

b) Sensor 2

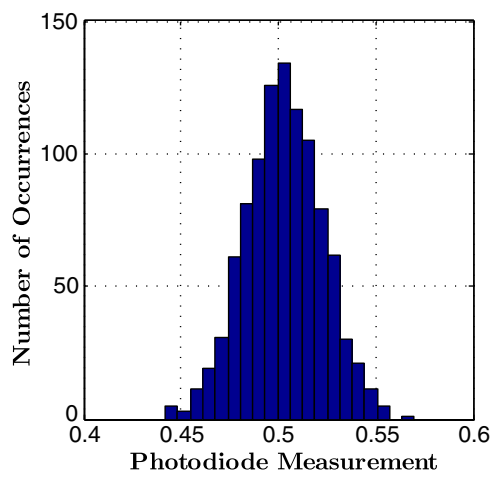

c) Sensor 3

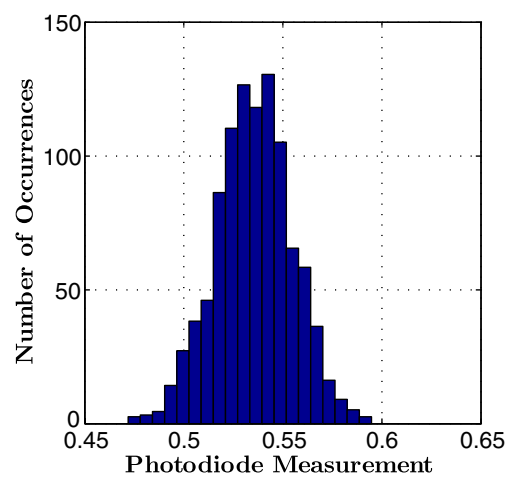

d) Sensor 4

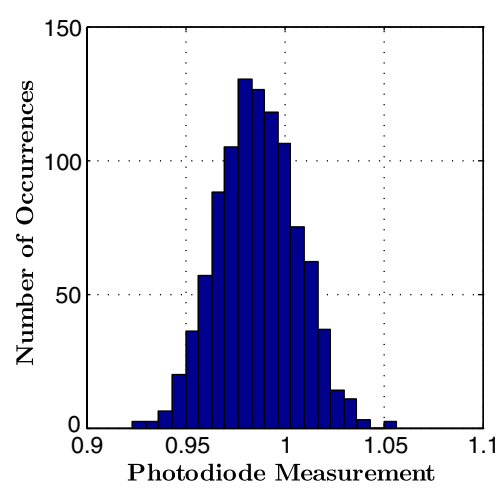

e) Sensor 5

Fig. A1 Histograms of the simulated measurements for the five sun sensors for which the simulated sun vector (direction on the attitude sphere) is in the field of view. The peak current output of the photodiodes is one and the standard deviation is 0.02 .

to meet requirements. This can result in both improved subsystem performance and reduced design time and cost.

\section{Appendix: Uncertainty of the Sun Vector Estimate}

The objective function used in the photodiode orientation optimization was derived using the covariance matrix of the sun vector estimate. In application to attitude determination, knowledge of the angular accuracy of the sun vector estimate is desired. However, because the error bounds on the components of the sun vector estimate form an ellipsoid and not a sphere, there is no single number quantifying the angular error that corresponds to the sun vector covariance matrix.

Two possible approximations of a single angular error corresponding to the sun vector covariance matrix are to use the radius of a sphere with equivalent volume as the error ellipsoid, or to use the semimajor axis of the error ellipsoid [16]. The former approximation is most accurate when the principal axes of the error ellipsoid are approximately equal, and the latter approximation is best when the semimajor axis of the error ellipsoid is much greater than the other two axes. Neither of these assumptions are generally valid for the optimization examples presented in this paper. To quantify the angular accuracy of the sun vector estimate without making any assumptions on the shape of the error ellipsoid, Monte Carlo simulations are performed to estimate the angular uncertainty resulting from a given photodiode configuration. The simulations consist of the following steps:

1) For each direction on the attitude sphere, calculate the ideal measurement of each photodiode.

2) For each trial, add pseudorandom numbers from a mean zero normal distribution to simulate the measurements. One thousand trials for each sun vector direction were used.

\footnotetext{
$\$$ This is under the assumption that the errors in the photodiode measurements have a Gaussian distribution.
}

3) Estimate the sun vector using the simulated measurements and constrained minimization of Eq. (9).

4) For each sun vector estimate, calculate the angular error using the dot product. That is,

$$
\delta \theta=\cos ^{-1}\left(\hat{\boldsymbol{s}}_{\mathrm{est}}^{T} \hat{\mathrm{s}}_{\text {true }}\right)
$$

where $\hat{\boldsymbol{s}}_{\text {est }}$ is the unit vector estimate and $\hat{\boldsymbol{s}}_{\text {true }}$ is the true unit sun vector.

5) The uncertainty in each sun vector direction is then taken to be the average angular uncertainty from the 1000 trials. For the $j$ th sun vector direction, this is denoted as $\bar{\delta} \theta_{j}$. This is the quantity shown in Figs. $\underline{8 b}, \underline{8 d}, 12 \mathrm{~b}$, and $12 \mathrm{c}$.

Example histograms resulting from the simulation for a single sun vector direction are shown in Figs. A1 and A2. Figure A1 shows the histograms of the photodiode measurements for all five photodiodes for which the sun was in the field of view for the given sun vector direction. The histogram of the resulting angular uncertainty is shown

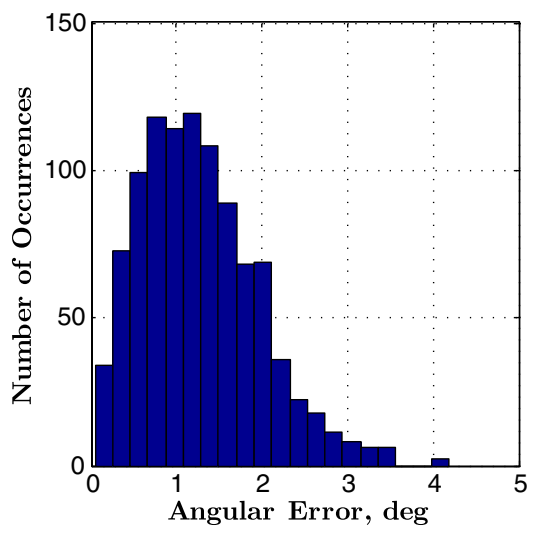

Fig. A2 Histogram of the angular error in the sun vector estimate. Mean error is $1.3 \mathrm{deg}$. 
in Fig. A2. These results are from the photodiode configuration of example 2 , optimized using identical weighting over the attitude sphere.

The angular uncertainty over the attitude sphere is taken to be the weighted average of the angular uncertainty in each sun vector direction on the sphere. That is,

$$
\delta \theta_{\text {total }}=\sum_{j=1}^{N}\left(\frac{W_{j}}{\sum_{k=1}^{N} W_{k}} \bar{\delta} \theta_{j}\right)
$$

This is the quantity given in Figs. $\underline{7 b}$ and $\underline{11 b}$.

\section{Acknowledgment}

This work is supported by the Department of Defense through a National Defense Science and Engineering Graduate Fellowship.

\section{References}

[1] Sidi, M. J., Spacecraft Dynamics and Control, a Practical Engineering Approach, Cambridge Univ. Press, Cambridge, England, U.K., 1997.

[2] Bhanderi, D. D. V., Spacecraft Attitude Determination with Earth Albedo Corrected Sun Sensor Measurements, Ph.D. Thesis, Aalborg Univ., Aalborg, Denmark, Aug. 2005.

[3] Springmann, J. C., Sloboda, A. J., Klesh, A. T., Bennett, M. W., and Cutler, J. W., "Attitude Determination System of the RAX Satellite," Acta Astronautica, Vol. 75, June-July 2012, pp. 120-135. doi:10.1016/j.actaastro.2012.02.001

[4] Taraba, M., Rayburn, C., Tsuda, A., and MacGillivray, C., "Boeing's CubeSat TestBed 1 Attitude Determination Design and On-Orbit Experience," Proceedings of the AIAA/USU Conference on Small Satellites, Utah State University, Paper SSC09-X-6, Aug. 2009.

[5] Ovchinnikov, M., Ilyin, A., Kupriynova, N., Penkov, V., and Selivanov, A., "Attitude Dynamics of the First Russian Nanosatellite TNS-0," Acta Astronautica, Vol. 61, Nos. 1-6, 2007, pp. 277-285. doi:10.1016/j.actaastro.2007.01.006

[6] Allgeier, S. E., Mahin, M., and Fitz-Coy, N. G., "Design and Analysis of a Coarse Sun Sensor for Pico-Satellites," Proceedings of the AIAA Infotech at Aerospace Conference and Exhibit and AIAA Unmanned... Unlimited Conference, AIAA Paper 2009-1837, April 2009.
[7] Reeves, E. I., "Spacecraft Design and Sizing," Spacecraft Mission Analysis and Design, Microcosm, Portland, OR, 1999, pp. 301-352.

[8] Jackson, B., and Carpenter, B., "Optimal Placement of Spacecraft Sun Sensors Using Stochastic Optimization,” 2004 IEEE Aerospace Conference Proceedings, IEEE Publ., Piscataway, NJ, March 2004, pp. 3916-3923.

[9] Mosher, T., "Spacecraft Design Using a Genetic Algorithm Optimization Approach," 1998 IEEE Aerospace Conference Proceedings, IEEE Publ., Piscataway, NJ, March 1998, pp. 123-134.

[10] Taylor, E., "Evaluation of Multidisciplinary Design Optimization Techniques as Applied to Spacecraft Design," 2000 IEEE Aerospace Conference Proceedings, Vol. 1, IEEE Publ., Piscataway, NJ, March 2000, pp. 371-384.

[11] Wertz, J. R., "Attitude Geometry," Spacecraft Attitude Determination and Control, Kluwer Academic, Norwell, MA, 1978, pp. 22-35.

[12] Tegmark, M., "Icosahedron-Based Method for Pixelizing the Celestial Sphere," Astrophysical Journal, Letters, Vol. 470, No. 2, Oct. 1996, pp. L81-L84. doi: $10.1086 / 310310$

[13] Teanby, N. A., "Icosahedron-Based Method for Even Binning of Globally Distributed Remote Sensing Data," Computers and Geosciences, Vol. 32, No. 9, Nov. 2006, pp. 1442-1450. doi:10.1016/j.cageo.2006.01.007

[14] Kjellberg, H. C., and Lightsey, E. G., "Constrained Attitude Control Module for Small Satellites," Proceedings of the 26th Annual AIAA/ USU Conference on Small Satellites, Utah State University, Paper SSC12-III-7, Aug. 2012.

[15] Lerner, G. M., "Sun Sensors," Spacecraft Attitude Determination and Control, Kluwer Academic, Norwell, MA, 1978, pp. 155-166.

[16] Lerner, G. M., "Covariance Analysis," Spacecraft Attitude Determination and Control, Kluwer Academic, Norwell, MA, 1978, pp. 429-434.

[17] Crassidis, J. L., and Junkins, J. L., Optimal Estimation of Dynamic Systems, CRC Press, Boca Raton, FL, 2004, pp. 62-84.

[18] Springmann, J. C., "On-Orbit Calibration of Photodiodes for Attitude Determination," Proceedings of the USU/AIAA Small Satellite Conference, Utah State University, Paper SSC13-VIII-1, Aug. 2013.

[19] Shuster, M. D., "Constraint in Attitude Estimation Part I: Constrained Estimation," Journal of Astronautical Sciences, Vol. 51, No. 1, Jan.March 2003, pp. 51-74.

[20] Cutler, J. W., and Bahcivan, H., "Radio Aurora Explorer-A Mission Overview," Journal of Spacecraft and Rockets, Vol. 51, No. 1, 2014, pp. 39-47. doi:10.2514/1.A32436 